\title{
The management of chronic breathlessness in patients with advanced and terminal illness
}

This Clinical Review (BMJ 2015;350:g7617, doi:10.1136/bmj. g7617) should have the volume number 350 (not 349 ). 